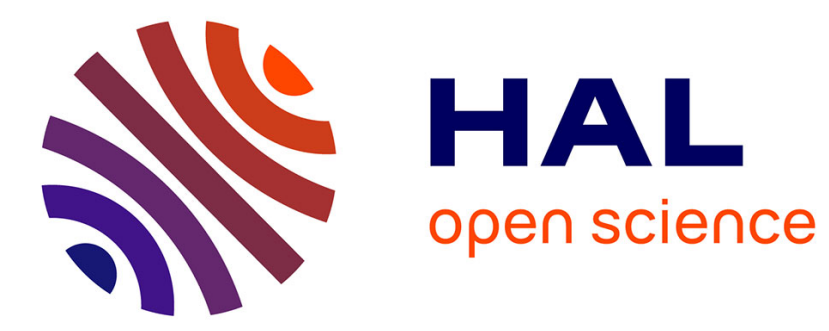

\title{
Psychometric Properties of the French Version of the Multifactorial Memory Questionnaire for Adults and the Elderly
}

Isabelle Fort, Linda Adoul, Delphine Holl, Joël Kaddour, Kamel Gana

\section{- To cite this version:}

Isabelle Fort, Linda Adoul, Delphine Holl, Joël Kaddour, Kamel Gana. Psychometric Properties of the French Version of the Multifactorial Memory Questionnaire for Adults and the Elderly. Canadian Journal on Aging / Revue canadienne du vieillissement, 2004, 23, pp.347 - 357. hal-01789129

\section{HAL Id: hal-01789129 \\ https://hal-amu.archives-ouvertes.fr/hal-01789129}

Submitted on 14 May 2018

HAL is a multi-disciplinary open access archive for the deposit and dissemination of scientific research documents, whether they are published or not. The documents may come from teaching and research institutions in France or abroad, or from public or private research centers.
L'archive ouverte pluridisciplinaire $\mathbf{H A L}$, est destinée au dépôt et à la diffusion de documents scientifiques de niveau recherche, publiés ou non, émanant des établissements d'enseignement et de recherche français ou étrangers, des laboratoires publics ou privés. 


\title{
Psychometric Properties of the French Version of the Multifactorial Memory Questionnaire for Adults and the Elderly
}

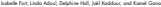

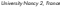

\begin{abstract}
thent

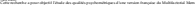

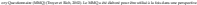

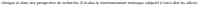

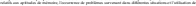

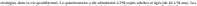

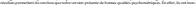

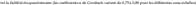

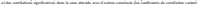

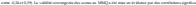

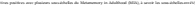

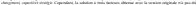

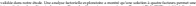

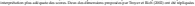

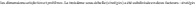

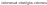

\begin{abstract}
vorer

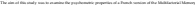

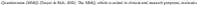

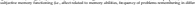

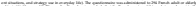

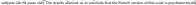

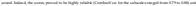

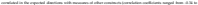

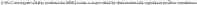

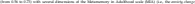

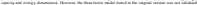

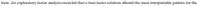

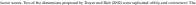

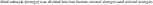

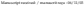

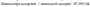

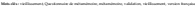

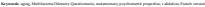

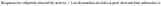

Foil Lame ikes

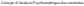

karese Manerd.

wis 10

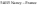

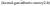

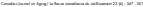




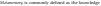

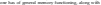

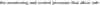

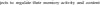

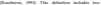

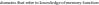

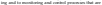

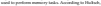

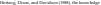

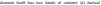

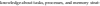

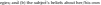

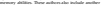

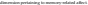

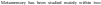

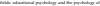

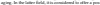

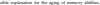

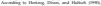

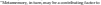

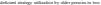

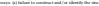

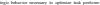

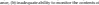

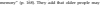

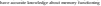

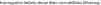

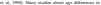

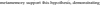

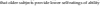

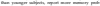

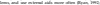

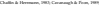

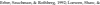

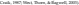

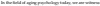

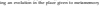

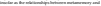

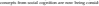

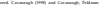

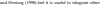

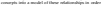

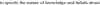

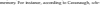

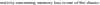

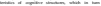

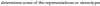

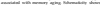

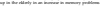

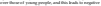

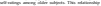

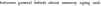

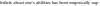

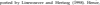

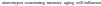

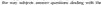

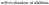

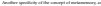

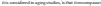

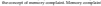

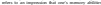

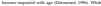

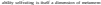

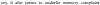

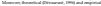

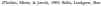

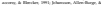

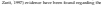

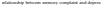

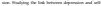

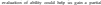

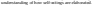

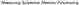

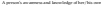

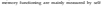

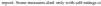

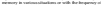

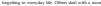

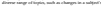

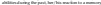

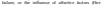

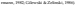

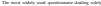

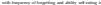

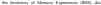

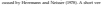

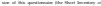

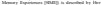

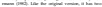

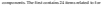

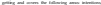

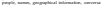

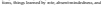

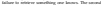

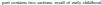

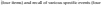

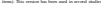

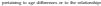

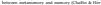

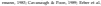

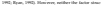

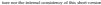
has bras ablhad

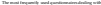

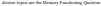

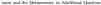

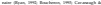

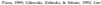




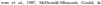

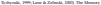

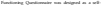

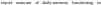

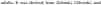

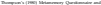

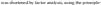

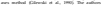

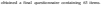

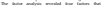

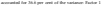

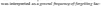

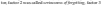

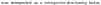

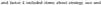

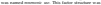

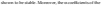

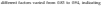

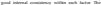

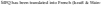

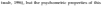

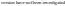

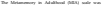

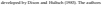

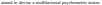

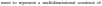

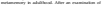

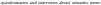

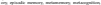

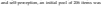

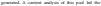

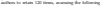

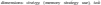

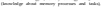

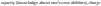

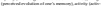

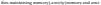

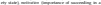

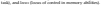

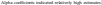

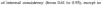

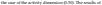

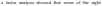

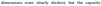

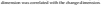

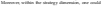

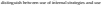

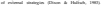

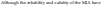

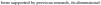

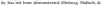

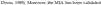

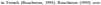

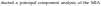

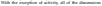

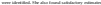

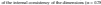

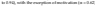

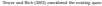

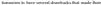

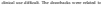

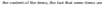

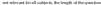

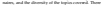

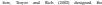

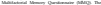

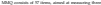

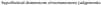

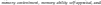

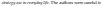

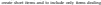

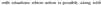

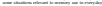

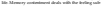

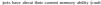

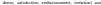

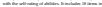

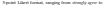

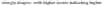

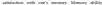

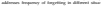

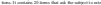

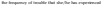

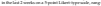

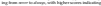

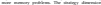

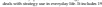

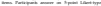

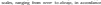

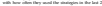

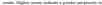

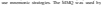

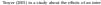

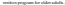

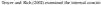

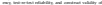

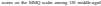

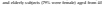

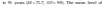

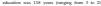

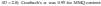

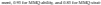

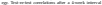

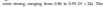

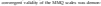

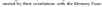

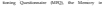

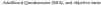

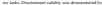

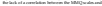




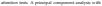

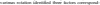

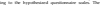

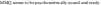
terevaliers

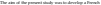

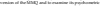

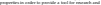

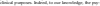

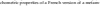

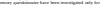

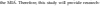

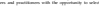

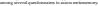

\section{Neftiol}

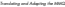

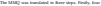

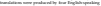

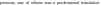

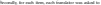

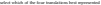

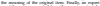

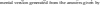

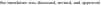

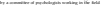

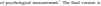

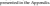

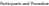

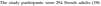

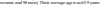

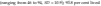

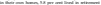

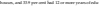

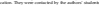

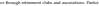

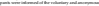

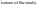

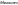

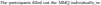

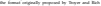

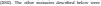

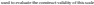

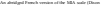

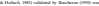

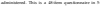

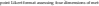

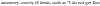

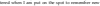

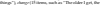

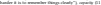

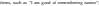

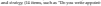

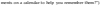

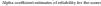

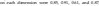
nowesity

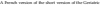

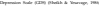

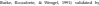

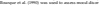

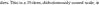

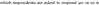

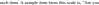

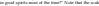

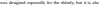

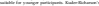

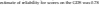

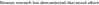

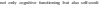

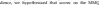

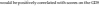

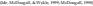

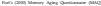

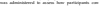

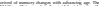

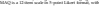

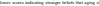

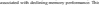

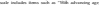

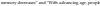

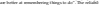

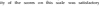

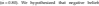

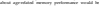

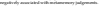

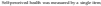

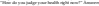

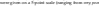

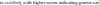

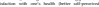
kidilat

\section{malk}

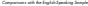

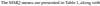

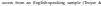

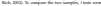

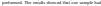

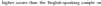

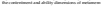

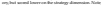

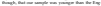

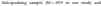

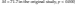




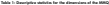

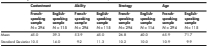

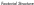

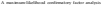

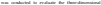

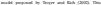

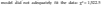

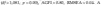

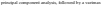

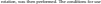

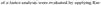

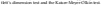

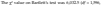

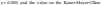

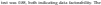

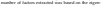

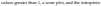

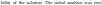

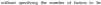

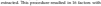

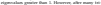

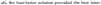

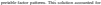

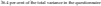

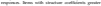

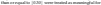
ito aturarain

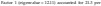

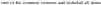

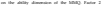

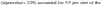

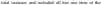

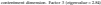

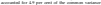

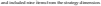

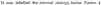

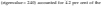

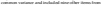

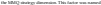

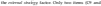

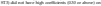
an later

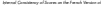

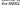

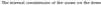

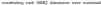

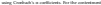

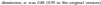

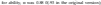

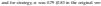
rat

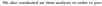

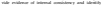

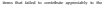

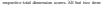

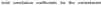

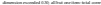

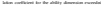

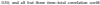

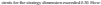

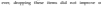

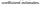

\section{Erenow Balkty}

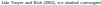

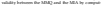

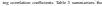

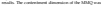

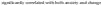

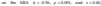

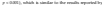

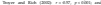

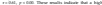

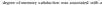

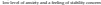

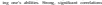

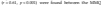

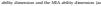

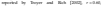

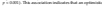

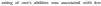

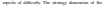

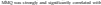

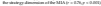




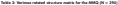

\begin{tabular}{|c|c|c|c|c|c|}
\hline \multirow[t]{2}{*}{ Trafn } & \multicolumn{4}{|c|}{ 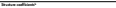 } & \multirow[t]{2}{*}{ Consentin: } \\
\hline & henere 1 & heima & hete 2 & Sorier a & \\
\hline TT & Wint & antr & -110 & $4 \pi$ & $\ln$ \\
\hline C: & kan & anis & Sich & घait & $10 \mathrm{sen}$ \\
\hline ca. & cave. & b.16s & osen & I 14 & wan \\
\hline$c$ & $13: 12$ & a.4h & $6: 14$ & wenk & tats \\
\hline ci. & axp & tatia & oser & yen & wan \\
\hline$C$ & kats & e.sta & $\operatorname{sen}$ & a) & $y=06$ \\
\hline Cr & wata & a.n & nese & asu & yen \\
\hline ct & ind & bata & bat & a.4 & bati \\
\hline $\cos$ & weis & II is & olat & an & yas \\
\hline cal & a.is & b.16 & bist & axts. & int \\
\hline $\mathrm{cHi}$ & tala & a.aka & 614 & asei & 1.411 \\
\hline $\operatorname{sis}$ & wate & a.t:4 & weta & a : 6 & yate \\
\hline cis: & มะแก & a.42 & s. & a ris & wath \\
\hline cla & $2 \pi s$ & e.415 & osat. & ants. & Eati \\
\hline cis & vas & 0.411 & Sxas & asta. & vals \\
\hline che & kat & a 400 & 6142 & a wa & wah \\
\hline ent & wat & e.sta & nis & Mavs & neso: \\
\hline cie & test & arrs & 614 & gah & $= \pm x$ \\
\hline \pm 1 & date & 1582 & So:kt & wan & wart \\
\hline 44 & eate & ist & $\Delta 1 \%$ & an & rath \\
\hline 4 & e.45 & 163 & ablo & มาแ & 14ar \\
\hline in & akis & nal & 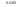 & aatr & laser \\
\hline ai & anda & and & oxw & ana & yat \\
\hline at & asia & a is & oan & ave & att \\
\hline st & Eais & waka & este & axr & Eabi \\
\hline $4 i$ & aseis & y $11 \mathrm{k}$ & osar & a nar & Eat1 \\
\hline \# & awa & wa & 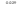 & cant & tene \\
\hline ate & e.4.2 & kate & Salst & axit & waw \\
\hline an & asta & bis & 6141 & Inith & Bact \\
\hline ats. & Gate & ma & osto & y.4 & 10.64 \\
\hline ats & anele & 1065 & siv & yaz & wau \\
\hline aid & Dask & tin & 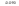 & aw & Wals \\
\hline
\end{tabular}

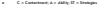

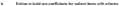


Shat 2 Can near

\begin{tabular}{|c|c|c|c|c|c|}
\hline \multirow[t]{2}{*}{ Provian } & \multicolumn{4}{|c|}{ 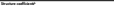 } & \multirow[t]{2}{*}{ 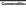 } \\
\hline & Femen 1 & Ineme 1 & haves : & Fante is & \\
\hline$\pi$ & tent & +17 & 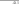 & 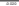 & $\sqrt{4}+2$ \\
\hline ats & a a a & 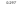 & a its & $\Delta \%$ & Q: $2=1$ \\
\hline$a r t$ & 4.24 & $=18$ & a $\sin$ & Sorate & 8208 \\
\hline$r=$ & 6. & 3a1s & a ila & Q61 & $\theta t=$ \\
\hline fat & e atis & in $1=$ & it $\operatorname{ses}$ & $-6 i \mathrm{~B}$ & 600 \\
\hline$\leq 28$ & s.a1 & atit & a in & $\Delta 1 \%$ & 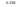 \\
\hline 41 & cast & sata & 4in & Sath & $\theta 20$ \\
\hline was & saik & $\cos 2$ & sitir & Salk & 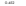 \\
\hline irs & sats & 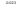 & an & xaka & $61 \mathrm{as}$ \\
\hline$M=$ & ine & $=18 x$ & $\operatorname{ESH}$ & sxas. & $\theta$ ane \\
\hline utr & Selve & alia & sitis & Sag & sat \\
\hline uts & 4 Not: & 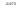 & a.atr & 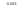 & otra \\
\hline urF & enos & eath & in 284 & S.424 & 820 \\
\hline ur & 4 ats & $a+6$ & a ast & $6: 2 y$ & ORen \\
\hline urp & Aint & a ik & $428 x$ & Gail & Q2:4a \\
\hline ura & 4711 & 4680 & 4118 & Guin & $\delta \mathrm{kin}$ \\
\hline urnt & detre & beta. & a ath & $61+4$ & $\phi=1$ \\
\hline eris & Gave & a $1 \mathrm{k}$ & it $\mathrm{zat}$ & sats & $\theta \mathrm{H}$ \\
\hline 1118 & a it & 4da & a sid & Q & $\theta=1$ \\
\hline nis & Sha & $=18$ & 4 eat & ob ar & \&UW \\
\hline urt: & ant & a ite & 4 ise & 6.a6s & Qtila \\
\hline uris & saik & A4a & s ats & 642 & 6201 \\
\hline ertF & seis & 4120 & 4 E⿰冫 & oint & Oulas \\
\hline whe & Gate & $\operatorname{sen}$ & a sut & Gath & $\theta=7$ \\
\hline art & Ga14 & atis & 4.48 & $624 a$ & 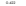 \\
\hline
\end{tabular}

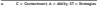

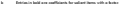

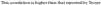

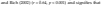

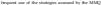

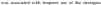

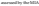

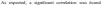

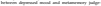

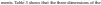

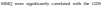

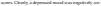

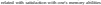

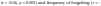

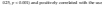

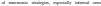

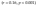

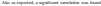

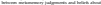

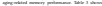

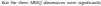

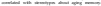

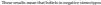

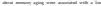

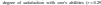

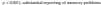




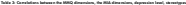

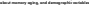

\begin{tabular}{|c|c|c|c|c|c|}
\hline & 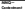 & whis-mity & lave-linang & 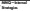 & 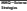 \\
\hline ind. Gimas: & & 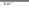 & & & \\
\hline bis. Gusara & Bant & & & & \\
\hline $\operatorname{sed} d a=4$ & Eis & & & & \\
\hline whe bonaty & & & 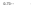 & aw- & orn \\
\hline 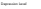 & sis & $4: 4$ & a ist & $m=-$ & $\Delta, 11$ \\
\hline 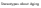 & $\mathrm{ax}$ & 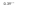 & $4 \times-$ & $a+k$ & an- \\
\hline $\ln 3$ & 411 & $\Delta 12$ & $\mathrm{EOH}$ & tis & Sil \\
\hline thanas & 504 & Q is & 416 & tiale & $\Delta \mathrm{BH}^{-}$ \\
\hline 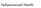 & in & $6: 2$ & $4 i x$ & 4.4 & at it \\
\hline
\end{tabular}

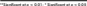

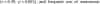

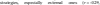
n rater

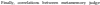

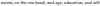

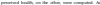

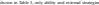

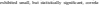

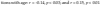

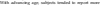

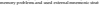

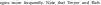

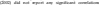

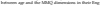

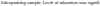

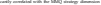

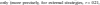

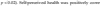

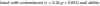

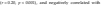

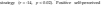

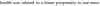

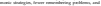

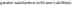

\section{biusues}

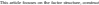

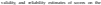

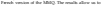

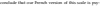

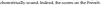

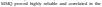

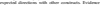

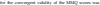

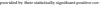

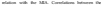

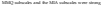

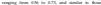

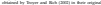

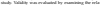

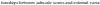

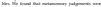

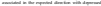

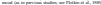

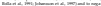

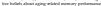

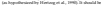

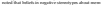

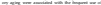

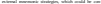

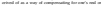

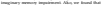

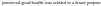

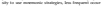

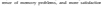

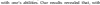

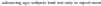

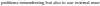

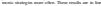

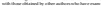

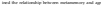

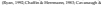

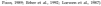

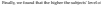

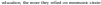

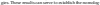

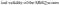

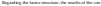
kestor la

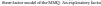




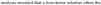

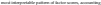

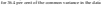

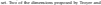

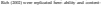

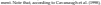

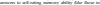

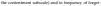

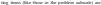

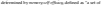

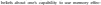

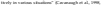

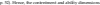

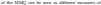

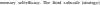

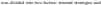

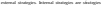

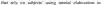

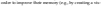

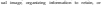

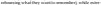

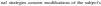

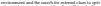

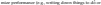

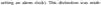

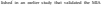

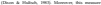

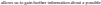

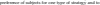

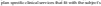
rolituming

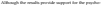

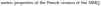

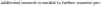

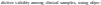

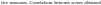

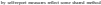

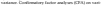

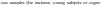

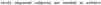

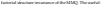

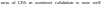

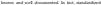

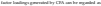

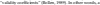

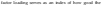

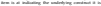
mist bi wermer

\section{inters}

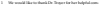
som

\section{8pheress}

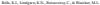

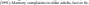

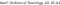

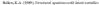

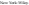

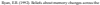

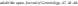

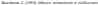

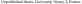

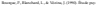

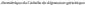

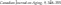

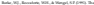

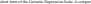

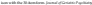

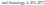

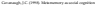

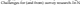

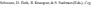

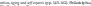
Fa miluing ho

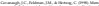

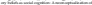

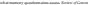
freturg icia a a

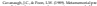

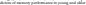

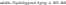

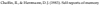

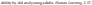
24

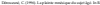

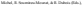

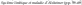

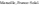

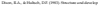

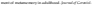
is $x=0 .+0$

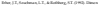

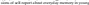

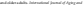

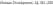

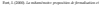

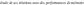

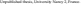

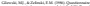

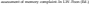

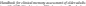

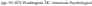
Pite a

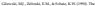

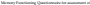

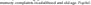

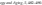

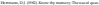

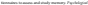

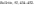




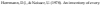

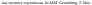

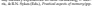

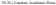

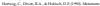

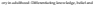

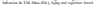

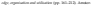

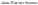

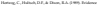

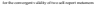

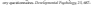
The

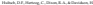

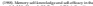

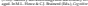

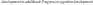

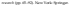

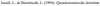

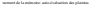

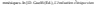

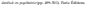

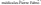

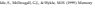

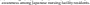

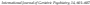

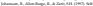

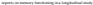

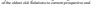

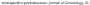
$1 \rightarrow 18$

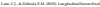

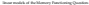

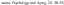

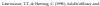

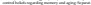

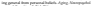

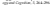

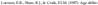

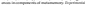

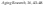

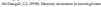

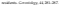

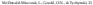

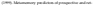

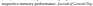

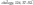

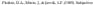

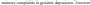

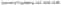

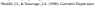

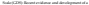

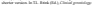

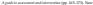

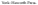

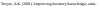

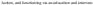

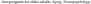

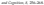

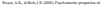

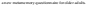

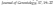

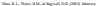

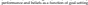

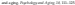

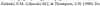

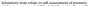

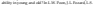

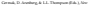

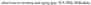

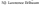

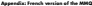

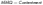

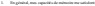

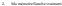

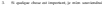
portablowe

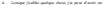

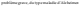

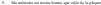

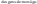

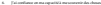

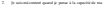
ingers

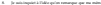

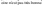

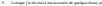

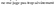

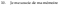

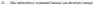

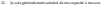
new

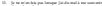

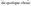

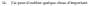

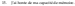

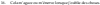

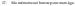

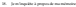

thate - Ahits

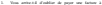
tentos

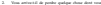

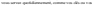
toreson 1

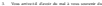

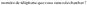




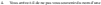

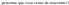

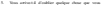

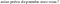

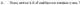

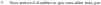

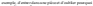
ruat have

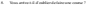

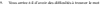

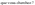

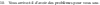

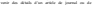

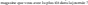

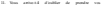

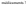

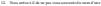

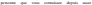
inesus

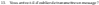

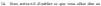

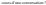

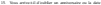

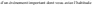

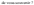

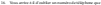

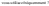

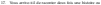

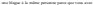

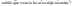

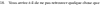

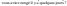

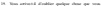

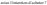

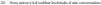
$\sin a$

\section{coves a alow}

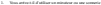

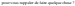

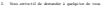

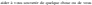

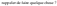

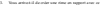

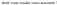

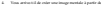

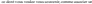

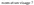

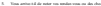

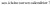

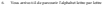

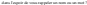

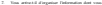

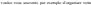

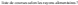

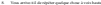

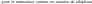

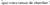

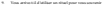

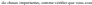

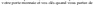
the seste

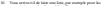

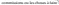

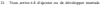

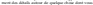

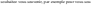

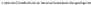

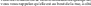

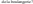

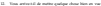

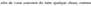

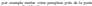

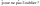

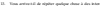

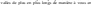
menerest

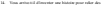

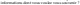

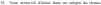

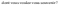

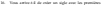

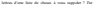

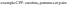

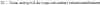

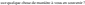

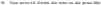

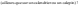

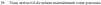

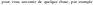

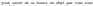
rothe ! 


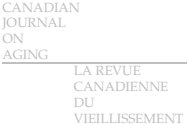

